\section{LETTER TO THE EDITOR}

missing from the HDS which has been of great value in my delirium research.

(6) As expected DSM-5 delirium was more common among medical inpatients than long-term care residents $(14.9 \%$ vs. $4.0 \%$, Table 1$)$.

(7) The Central Coast Australia Delirium Intervention Study (Regal, 2015) supported the gold standard to prove delirium was the speed and amplitude of both onset and recovery. In CADIS, the mean recovery of five-digit span forward was two days, six-DSF 5.6 days and Delirium Index 8.0 days. a decision by medical specialists. When the person has prior dementia, identifying delirium is a high-level medical skill. Behavioral and psychological symptoms of dementia can closely mimic delirium unless the examiner focuses on behavioral triggers and the speed of cognitive decline.

(2) The requirement of taking medications twice a day may have eliminated some delirium patients with impaired swallowing or refusal to take medications.

(3) Lumping dementia, confusion, and disorientation into one label "cognitive impairment" is problematic and reduces the usefulness of Table 3.

(4) The authors used prior diagnosis of dementia as a gold standard when in fact many people with that label have mild cognitive impairment.

(5) The ten "concentration" subtests in the Hierarchic Dementia Scale (HDS) (Cole and Dastoor, 1987) are not purely indices of concentration. Serial seven subtraction is impaired in low education with normal concentration. Months of the year backwards is often ranked as both an attention and executive function test and it requires semantic knowledge. Digit span forward is a rapid attention test

\section{Inattention tests for delirium: reply to Paul Regal}

I would like to thank Dr Regal for his interest in our work. He has some questions about our study that I am happy to respond to here.

To answer his first question concerning the research assistants' (RA) training, they were trained in lectures and coaching sessions to use the HDS and the CAM. DSM-5 diagnosis criteria were applied after their assessments. This is known to be an appropriate and more efficient way of proceeding than a single evaluation by a medical specialist (Zou et al., 1998).

His second comment concerned the requirement that participants be taking medications at least twice a day. It is true that this requirement may have led to the exclusion of $1 \%$ of potential participants. This percentage is very low because in our clinical settings, all patients, even those that have trouble swallowing, take medications. Pills are given with apple sauce or other food. And so this requirement cannot have had any real impact in this study. Refusal to take medication happens only occasionally in our clinical settings and the nursing staff are usually able to cope with this challenge. If this problem had any importance in our research, we would have taken measures to deal with it. It is worth noting, however, that when RADAR was created we assessed these issues directly, in 
order to test the feasibility of implementing this tool.

In response to Dr Regal's third comment, it must be said that unfortunately, the label "cognitive impairment" reveals the reality of the quality of the medical documentation in most clinical settings throughout the world. Since many medical specialists do not feel comfortable making a diagnosis of dementia (now termed major neurocognitive disorder), they often rely on this designation to indicate the presence of memory problems in the medical chart. The ideal way to obtain a proper diagnosis would be to have welltrained medical specialists evaluate every patient, something that is rarely possible, especially given the limited financial resources for doing research.

As to the fourth comment, in Canada, diagnosis of dementia is made at a late stage of the disease rather than at the beginning. Under-detection of dementia appears to be a problem worldwide (http://www.alz.co.uk/research/ world-report-2011). Simply put, we fail to understand why many patients with dementia in our study would have mild cognitive impairment instead of dementia.

To answer the fifth comment, the ten items related to attention in the HDS are bedside tests regularly used in clinical practice and research. We agree that they certainly cannot be interpreted purely as measures of attention. In fact, clinicians cannot rely on one single test to be certain that a patient has an attention problem; not even the Digit span forward can guarantee this. To ascertain whether or not there is an attentional problem, it is best to rely on professional neuropsychologists. However, professional assessments would involve spending far more time than is possible in this research, to determine the presence of such a problem.

Regarding the sixth comment, we have known for some time that onset and recovery is a great way to measure delirium (http://www.rima.org/web/ medline_pdf/jamgeriatrsoc_867-70.pdf). However, our study was a secondary analysis research based on cross-sectional data, so it was impossible to test for any change in the cognitive scores with the HDS.

\section{Conflict of interest}

None.

\section{Reference}

Zou, Y., Cole, M. J., Primeau, F. J., McCusker, J., Bellavance, F. and Laplante, J. (1998). Detection and diagnosis of delirium in the elderly: psychiatrist diagnosis, confusion assessment method, or consensus diagnosis?. International Psychogeriatrics, 10, 303-308. 